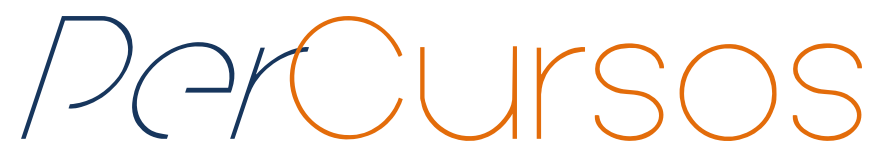

\title{
Resenha da exposição digital "The origins of the Internet in Europe" - O mundo em rede: contribuições de Paul Otlet e Henri La Fontaine
}

MUNDANEUM. The origins of the Internet in Europe: collecting, indexing \& sharing knowledge. Exposição digital. [Projeto Google Arts \& Culture]. 2013. Disponível em: <https://www.google.com/culturalinstitute/beta/exhibit/QQ-RRhoA?hl=pt-BR>. Acesso 19 set. 2016.

\author{
Igor Soares Amorim \\ Doutorando em Ciência da Informação na \\ Universidade Federal de Santa Catarina - UFSC. \\ Brasil \\ amorim.igors@gmail.com \\ Renata Cardozo Padilha \\ Doutoranda em Ciência da Informação na \\ Universidade Federal de Santa Catarina - UFSC. \\ Brasil \\ renatapadilha@hotmail.com
}

\section{Para citar esta resenha:}

AMORIM, Igor Soares; PADILHA, Renata Cardozo. Resenha da exposição digital "The origins of the Internet in Europe" - O mundo em rede: contribuições de Paul Otlet e Henri La Fontaine. Revista PerCursos. Florianópolis, v. 17, n. 34, p. 141 - 146, maio/ago. 2016.

DOI: $\mathbf{1 0 . 5 9 6 5 / 1 9 8 4 7 2 4 6 1 7 3 4 2 0 1 6 1 4 1}$

http://dx.doi.org/10.5965/1984724617342016141 
Os efeitos socioculturais gerados a partir do entrelaçamento das máquinas eletrônicas de base binária nas rotinas das pessoas, coletivos, empresas e Estados é designado por cultura digital, a qual diz respeito aos artefatos e aos sistemas de significação e comunicação. Para Gere (2008), a tecnologia digital é resultado da cultura digital, o que implica numa introjeção da subjetividade digital nas máquinas que já estava presente nos homens. A máquina social precede a máquina técnica e, na cultura digital, as subjetivações-modelos são a abstração, a codificação, a autorregulação, a virtualização e a programação (GERE, 2008). Em grande medida, a cultura digital foi impulsionada pelos sonhos de Paul Otlet e La Fontaine, que hoje se concretizam com a internet ${ }^{1}$.

A partir disso, apresentamos uma resenha da exposição digital The origins of the Internet in Europe (2013), criada pelo Mundaneum e disponibilizada na plataforma Google Arts \& Culture ${ }^{2}$, cuja proposta é apresentar a trajetória dessa dupla que desejava catalogar a produção intelectual do mundo. Paul Otlet e Henri La Fontaine "prenunciaram a rede de conhecimento que um século mais tarde se tornou a Internet com seus motores de busca!" (MUNDANEUM, 2013, tradução nossa).

O Mundaneum localizado em Mons, na Bélgica, desde 1998 abriga grande parte do patrimônio documental relacionado com os projetos de Paul Otlet e Henri La Fontaine. Em 2012, Mundaneum e Google realizam uma parceria na qual a Instituição passa a ser reconhecida como o "Google de papel” (MUNDANEUM, 2013, tradução nossa).

A exposição apresenta por meio de textos e imagens, que reproduzem os objetos das coleções do Mundaneum, a construção da rede mundial de coleta, indexação e compartilhamento de informação e conhecimento projetado por Otlet e La Fontaine. The origins of the Internet in Europe (2013) é composta por 38 imagens que reproduzem fotografias, documentos escritos e ilustrações; três vídeos que expõem conteúdos

\footnotetext{
1 Em World Science Festival (2012), que aconteceu em Nova lorque, Otlet foi reconhecido como "pai da ideia de internet" (MUNDANEUM, 2013, tradução nossa).

${ }^{2}$ Este é um projeto do Google Cultural Institute, um serviço especializado da empresa Google criado em 2011, cujo objetivo é disponibilizar via rede os bens culturais salvaguardados nos museus e instituições afins das várias regiões do mundo.
} 
distintos sobre a temática proposta: o primeiro, apresenta a visita de Andrew Carnegie 3 ao "Palais Mondial-Mundaneum" em Bruxelas; o segundo, trata-se de uma entrevista com o professor Boyd Rayward, biógrafo de Paul Otlet; e o terceiro, exibe a fala do jornalista Alex Wright sobre os novos desafios da informação. Além desses recursos expográficos, são apresentados textos informativos ao longo da exposição sobre Otlet, La Fontaine, CDU e o projeto Mundaneum em detalhes. Compreende-se que a exposição apresenta sua temática fundamentalmente por meio de recursos imagéticos que reproduzem os objetos autênticos dos criadores do projeto Mundaneum.

A exposição inicialmente apresenta as ambições e pontua algumas realizações, como a criação de um centro internacional de documentação e o desenvolvimento de instrumentos para o tratamento de documentos. Uma das primeiras imagens apresenta a capa da maior obra de Paul Otlet, Traité de Documentation. le Livre sur le livre (1934)4 O documentalista belga propôs uma série de instrumentos e concepções para manipular o conteúdo dos documentos, que de certa maneira anteciparam as tecnologias web. Ele vislumbrava um sistema de conexão e de acesso remoto a qualquer documento, desde que previamente tratado pelo centro universal de documentação.

Conforme a exposição (MUNDANEUM, 2013, tradução nossa), duas questões orientavam os trabalhos de Otlet e La Fontaine: “O que tem sido escrito por um autor?" e “O que tem sido escrito sobre um assunto?”. A fim de dar conta da produção intelectual do mundo, a dupla belga elaborou técnicas e tecnologias para indexar e organizar o conhecimento, uma delas é a Classificação Decimal Universal (CDU)5 editada em 1905 sob o título Manuael du Répertoire Bibliographique, sendo renomeada em sua segunda edição em 1925.

A exposição exibe o desenho ${ }^{6}$ da rede que conectaria os usuários a um centro mundial de documentação por meio de uma tela com um telefone, onde os conteúdos

\footnotetext{
${ }^{3}$ Empresário e filantropo estadunidense tornou-se um investidor dos projetos de Otlet e La Fontaine.

${ }^{4}$ https://www.google.com/culturalinstitute/beta/asset/trait\%C3\%Ag-de-documentation-le-livre-sur-lelivre/XwHXH_LywTGaQ

${ }^{5}$ https://www.google.com/culturalinstitute/beta/asset/the-universal-decimal-classification/7gFoJRt1fOA5VA

${ }^{6}$ https://www.google.com/culturalinstitute/beta/asset/documentation-and-telecommunications/1wFRAQsathoXw
} 
dos livros seriam acessados a distância: "Ao longe, um imenso edifício contendo todos os livros e cada pedaço de informação. As perguntas seriam feitas por telefone, e a página com a resposta é mostrada na tela para ser lida" (MUNDANEUM, 2013, tradução nossa). Em complemento, a exposição afirma que Otlet era um homem à frente de seu tempo, destacando sua ideia de rede, expondo reproduções do Tratado de Documentação com projetos tecnologicamente ambiciosos ${ }^{7}$ e descrevendo sua concepção sobre um sistema de hipertexto analógico (MUNDANEUM, 2013).

Ao fim da Primeira Guerra Mundial, Otlet e La Fontaine reforçaram a ideia de que cooperação intelectual internacional poderia conduzir à paz. Desde 1910 Otlet maturava a ideia de uma Cidade Mundial $^{8}$, dedicada à coleta, preservação, tratamento e disseminação do conhecimento, porém o projeto não saiu do papel.

Assim, a exposição segue para o fim, com fotos do atual Mundaneum, de seus arquivos $^{9}$ e espaço ${ }^{10}$, fechando num vídeo seguido do texto:

Nesta era de transmissões digitais e Web 2.0, a consciência de novos desafios relacionados não só para organizar e compartilhar conhecimento, mas também para a cidadania está começando a emergir. A essência do Mundaneum é como ele empresta profundidade e perspectiva a esta revolução digital, o que estamos a observar a partir de um ponto de vista privilegiado... (MUNDADENUM, 2013, tradução nossa)

No que tange os aspectos da organização e representação da informação ${ }^{11}$ na exposição resenhada, verificamos que os objetos são descritos com até quatro metadados ${ }^{12}$, sendo eles: título, data, local e external link, sendo este último um hiperlink para a página do Mundaneum. Constatamos que este conjunto de descritores reduz a

\footnotetext{
${ }^{7}$ https://www.google.com/culturalinstitute/beta/asset/documentation-andtelecommunications/7gG81qF6PAHs3A

${ }^{8}$ https://www.google.com/culturalinstitute/beta/asset/an-international-world-center/lgGe8NJ2jsZLfO

${ }^{9}$ https://www.google.com/culturalinstitute/beta/asset/the-mundaneum-s-archives/BgH4dhm52dH3eg

${ }^{10} \mathrm{https://www.google.com/culturalinstitute/beta/asset/the-museum-mundaneum/1QGNvFoM324g6w}$

${ }^{11}$ Refere-se ao processo que proporciona ao indivíduo o acesso e a recuperação da informação e, com isso, a possibilidade de produzir conhecimento (BRÄSCHER; CAFÉ, 2010).

${ }_{12}$ Trata-se de dados que se referem a outros dados, a fim de descrever as informações sobre forma e conteúdo de um objeto/documento que é fonte de informação.
} 
possibilidade informacional que os objetos reproduzidos imageticamente possuem, dificultando o acesso e a recuperação da informação sobre os objetos expostos.

Otlet compreendia as possibilidades de articulação entre a máquina social e a máquina técnica. Com La Fontaine, buscava a criação de uma rede de documentação, sustentada por diversas tecnologias (muitas das quais precisavam ser inventadas) instituída no centro de uma Cidade Mundial a fim de alcançar a paz por meio da cooperação intelectual entre as nações.

As contribuições otletianas têm sido retomadas a fim de repensar os fenômenos de informação e conhecimento a partir de um ponto de vista simbólico, considerando que tais fenômenos se constituem nas relações sociais e em contextos específicos. O enfoque atualizado se aplica sobre os estudos de informações algorítmicas e analógicas. A dimensão simbólica implica na consideração dessa nova cultura e nas subjetivações nos artefatos, nos sistemas de significação e comunicação e nos sujeitos. Em meio às liberdades e as rizomáticas formas de controle, o legado de Otlet torna-se referência para pensar o potencial da cultura digital (DAY, 2016).

Nesse contexto, a exposição digital revela-se uma fonte de informação importante para a produção de conhecimentos, no âmbito da cultura digital. Assim, ela própria tornase um documento que necessita ser processado e pensado informacionalmente, por meio dos estudos de organização e representação da informação e do conhecimento.

\section{Referências}

BRÄSCHER, M.; CAFÉ, L. Organização da informação ou organização do conhecimento? In: LARA, Marilda L.G.; SMIT, Johanna. (Org.). Temas de pesquisa em ciência da informação no Brasil. São Paulo: Escola de Comunicação e Artes/USP, 2010. p. 85-103.

DAY, Ronald. Todo lo que es pertinente: documentos e indizabilidad. Scire: representación y organización del conocimiento, v. 22, n. 1, p. 57-63, 2016. Disponível em: < http://www.ibersid.eu/ojs/index.php/scire/article/view/4304>. Acessado em: 19 set. 2016.

GERE, Charlie. Digital Culture. 2.ed. Londres: Reaktion Books, 2008. 
Resenha da exposição digital "The origins of the Internet in Europe” - O mundo em rede: contribuições de Paul

Recebida em: 19/09/2016 Aprovada em: 20/09/2016

Universidade do Estado de Santa Catarina - UDESC Centro de Ciências Humanas e da Educação - FAED

Revista PerCursos

Volume 17 - Número 34 - Ano 2016 revistapercursos@gmail.com 